\title{
Platypnoea-Orthodeoxia Syndrome: An Intriguing Diagnosis
}

\author{
Vânia Rodrigues $^{1}$, Tiago Fernandes Gomes ${ }^{2}$, Adriana Santos Silva ${ }^{1}$, Rita Rocha $^{1}$, Ana Ferrão ${ }^{1}$ \\ ${ }^{1}$ Internal Medicine Department, Centro Hospitalar de Leiria, Leiria, Portugal \\ ${ }^{2}$ Dermatology Department, Centro Hospitalar de Leiria, Leiria, Portugal
}

Received: 20/12/2018

Accepted: 09/01/2019

Published: 22/02/2019

\begin{abstract}
How to cite this article: Rodrigues V, Fernandes Gomes T, Santos Silva A, Rocha R, Ferrão A. Platypnoea-orthodexia syndrome: an intriguing diagnosis.
\end{abstract} EJCRIM 2019;6: doi:10.12890/2019_001030.

Conflicts of Interests: The Authors declare that there are no competing interests.

This article is licensed under a Commons Attribution Non-Commercial 4.0 License

\section{ABSTRACT}

Platypnoea-orthodeoxia syndrome (POS) is an uncommon clinical entity characterized by dyspnoea and hypoxaemia induced by upright posture and relieved by recumbence. It is often associated with right-to-left shunting through a patent foramen ovale (PFO) or an atrial septal defect. We report the case of a 79-year-old woman with hypoxaemia initially attributed to a pulmonary infection but persisting after successful treatment. Being in the upright position triggered the hypoxaemia. A thoracic CT angiogram and ventilation/perfusion lung scan excluded a pulmonary embolism, but a transoesophageal echocardiogram with a bubble test showed a PFO with a right-to-left shunt, without pulmonary hypertension. The patient underwent percutaneous closure of the PFO which led to prompt symptom relief and full functional recovery.

\section{LEARNING POINTS}

- Platypnoea-orthodeoxia syndrome is an uncommon condition that should be suspected in the presence of unexplained positional hypoxaemia.

- A high level of suspicion is required and the diagnosis can be safely established by tilt-table transoesophageal echocardiography.

- Percutaneous closure of a patent foramen ovale is safe and effective even in elderly patients.

\section{KEYWORDS}

Hypoxaemia, patent foramen ovale, dyspnoea, platypnoea-orthodeoxia

\section{CASE DESCRIPTION}

We report the case of a 79-year-old female patient with a history of pulmonary embolism, transient ischaemic attack and hypertension, who was medicated with a direct oral anticoagulant and who presented to the emergency department due to sudden onset of dyspnoea and dry cough. She denied chest pain, fever, palpitations or other symptoms. On observation the patient had cyanosis and mild hypoxaemic respiratory failure, and was admitted with the diagnosis of acute tracheobronchitis. However, the hypoxia persisted and was increased in orthostatism even after the infection had completely resolved.

\section{METHODS AND PROCEDURES}

Blood gas analysis revealed type 2 respiratory failure. Serial D-dimer levels and a chest X-ray showed no significant changes, so a thoracic CT angiogram was performed but did not suggest a pulmonary embolism. In order to exclude other conditions, an abdominal and pelvic CT scan was ordered but did not show any hepatic disease. Because of the possibility of a chronic peripheral pulmonary embolism, a ventilation/perfusion lung scan was performed and showed an anomalous uptake of albumin macroaggregates labelled with technetium 
$(99 \mathrm{mTc})$ sestamibi in the kidneys and brain, revealing the existence of a right-to-left shunt. In light of these findings, a transoesophageal echocardiogram and a bubble test with administration of agitated saline through a peripheral vein was requested and showed a displaced interatrial septum and the presence of a patent foramen ovale (PFO) with passage of agitated saline serum into the left atrium, even without the Valsalva manoeuvre and without pulmonary hypertension.

Subsequently, the patient underwent percutaneous closure of the PFO with a $30 \mathrm{~mm}$ AtriaSept device. The echocardiogram after the intervention revealed no evidence of residual right-to-left shunting. Blood gas analysis was performed and as the patient no longer had hypoxia on recumbency or orthostatism, she was discharged with scheduled follow-up consultations in Internal Medicine and Cardiology. Clinical follow-up has been favourable with no new desaturation episodes and the patient has returned to her normal life.

\section{DISCUSSION}

PFO is a congenital cardiac condition that allows communication between the right and left heart cavities and is present in about $25 \%$ of individuals. Although it is usually an occasional finding with no haemodynamic repercussions, it can be associated with paradoxical embolism, stroke, transient global amnesia, migraine headache and platypnoea-orthodeoxia syndrome (POS) ${ }^{[1]}$.

POS is a rare condition characterized by dyspnoea and hypoxaemia triggered by orthostatism and relieved by lying in the supine position ${ }^{[2]}$. This entity was first reported by Burchell in 1949 and more than 180 cases have since been described in the literature. Most cases present in the elderly, as aging may affect the size of a PFO, as it is also linked to aortic root dilation, distortion of the interatrial septum and stiffness of the right ventricle ${ }^{[3]}$.

The precise pathophysiology of POS is still unknown, but three mechanisms have been suggested: cardiac conditions with intracardiac shunting, pulmonary conditions with ventilation/perfusion mismatch, and hepatic diseases such as hepatopulmonary syndrome (HPS) which causes intrapulmonary vascular dilatation, interatrial communication being the most common aetiological association ${ }^{[4]}$. Our patient did not show any evidence of pulmonary or hepatic disease so we looked for a cardiac condition.

A transoesophageal echocardiogram together with intravenous injection of agitated saline solution usually confirms the diagnosis and reveals more accurately the right-to-left shunt that occurs with orthostatism if performed on a tilt table. In parallel with imaging, serial positional oxygen measurements should be taken to estimate shunt magnitude. The gold standard for the diagnosis of POS related to intracardiac shunting remains cardiac catheterization with direct measurement of oxygen saturation in the left atrium and pulmonary veins; however, in our daily clinical practice, non-invasive work-up is usually enough ${ }^{[4,5]}$. In the present case we found a clear anatomical defect, that is, a PFO confirmed by transoesophageal echocardiography with a bubble test.

The definitive treatment of patients with POS associated with atrial shunting is closure of the atrial defect with a device, either surgically or via a percutaneous approach. Percutaneous closure has been shown to be effective in patients of all ages, avoids the mortality and morbidity associated with open-heart surgery, and is associated with total resolution of the shunt, as was the case in our patient. The decision to treat should be guided by patient disability rather than shunt magnitude ${ }^{[1,5]}$.

\section{REFERENCES}

1. Rodrigues P, Monteiro M, Palma P, Sousa-Pereira L, Cabral S, Oliveira F, et al. Body position and oxygenation: an intriguing relationship. Rev Port Cardiol 2014;33:385.e1-5.

2. Takhar R, Biswas R, Arora A, Jain V. Platypnoea-orthodeoxia syndrome: novel cause for a known condition. BMJ Case Rep 2014;2014:bcr2013201284.

3. Sanikommu V, Lasorda D, Poornima I. Anatomical factors triggering platypnea-orthodeoxia in adults. Clin Cardiol 2009;32:E55-57.

4. Madeira S, Raposo L, David R, Marques A, Gomes JA, Cardim N, et al. Dyspnea in a nonagenarian: the usual suspects, an unexpected culprit. Rev Port Cardiol 2015;34:559.e1-6.

5. Queirós C, Francisco E, Almeida J. Platypnea-orthodeoxia syndrome after complicated cholecystectomy: an unsuspected diagnosis. Acta Med Port 2017;30:827-830. 
European Journal

of Case Reports in

Internal Medicine 